\title{
Digital and physical prototype of an Asymptotic Facade Module.
}

\author{
Eike Schling*, Zongshuai Wan ${ }^{\dagger}$ \\ * The University of Hong Kong \\ Faculty of Architecture \\ Department of Architecture \\ Knowles Building, KB413, Pokfulam Road, Pokfulam, Hong Kong \\ Email: schling@hku.hk, web page: https://www.arch.hku.hk/staff/arch/schling-eike/ \\ † Email: wanzongshuai@gmail.com
}

\begin{abstract}
Form-active structures enable a spatial load transfer via compression and tension, and thus allow for an optimal use of material to create lightweight, transparent building envelopes. Nonetheless, their application in architecture remains rare and specialized, as their free-form geometry creates high costs in the fabrication and assembly of individual and spatially complex parts, and often lacks to integrate with the built environment.

We present current research on low-cost, high-performance doubly curved building envelopes that benefit from a curvilinear layout along an isothermal web on minimal surfaces. We investigate the geometric symbiosis of an elastic lamella mullion system that follows the asymptotic curves combined with panelization strategies along the principal curvature lines in order to simplify fabrication and enhance the structural performance.
\end{abstract}

We created a digital and physical setup of a prototypical asymptotic façade substructure of $2.4 \times 2.4 \mathrm{x}$ $2.4 \mathrm{~m}$, in collaboration with the construction industry (GOMORE, Taiwan). We evaluate the complete design and construction process, including digital modelling, fabrication planning, computer-aided manufacturing and logistics, prefabrication, and assembly. Our focus lies on a novel construction process, which embeds the geodesic curvature within the composite lamellas during prefabrication, and introduces the geodesic torsion during the elastic assembly process. We use the physical prototype to verify our digital simulations.

Finally, both digital and physical prototypes are used to investigate cladding solutions. Our studies are specifically looking at developable-elastic and planar-rigid tessellations, which utilize a principal curvature layout. We conclude by highlighting flat-sheet fabrication, high structural resilience, and assembly time and tolerances, as primary potentials and challenges of this design strategy.

Keywords: Asymptotic Curves, Principal Curvature Line, Double Curvature, Building Envelope, Elastic Construction

\section{REFERENCES}

[1] Pottmann, Helmut; Schiftner, Alexander; Bo, Pengbo; Schmiedhofer, Heinz; Wang, Wenping; Baldassini, Niccolo; Wallner, Johannes (2008): Freeform surfaces from single curved panels. In : ACM SIGGRAPH 2008. ACM SIGGRAPH 2008. Los Angeles, California, 11.08.2008 - 15.08.2008. New York, New York, USA: ACM Press, 76: 1-10.

[2] Schling, Eike (2018): Repetitive Structures. Design and Construction of Curved Support Structures with Repetitive Parameters. Dissertation, Chair of Structural Design, Faculty of Architecture, Technical University of Munich. DOI: 10.14459/2018md1449869

[3] Schling, Eike; Hsu, Chih Lin; Ma, Muye (2021): Asymptotic building envelope. Combining the benefits of asymptotic and principal curvature layouts. With assistance of GOMORE. In Anastasia Globa, Jeroen van Ameijde, Adam Fingrut, Nayeon Kim, Tian Tian Sky Lo (Eds.): Projections. Proceedings of the 26th International Conference of the Association for Computer-Aided Architectural Design Research in Asia. CAADRIA 2021, vol. 1. Hong Kong, pp. 341-350. 